\title{
Thanks to Editorial Board of Arquivos de Neuro-Psiquiatria
}

The Editors of Arquivos de Neuro-Psiquiatria thanks to Associate Editors (66), Editorial Board (52) and Referees (538) who altruistically and without payment have expended their time to develop the editorial tasks of this journal since January 1st, 2020 to December 31st, 2020

Thank you very much!

\section{ASSOCIATE EDITORS}

Adriana Bastos Conforto

Adriana Moro

Alexandra Prufer Queiroz Campos Araújo

Ana Carolina Coan

Analuiza Camozzato

Antonio José da Rocha

Antonio Lucio Teixeira

Ayrton Roberto Massaro

Carlos Otto Heise

Carolina de Medeiros Rimkus

Celi dos Santos Andrade

Chien Hsin Fen

Clarissa Lin Yasuda

Cristiane Nascimento Soares

Dalva Lucia Rollemberg Poyares

Daniel Ciampi de Andrade

Edmar Zanoteli

Eduardo Genaro Mutarelli

Elisa de Paula França Resende

Ethel Mizrahy Cuperschmid

Fábio A. Nascimento

Fabíola Dach
Francisco de Assis Aquino Gondim Gabriel Rodriguez de Freitas Grace Schenatto Pereira Moraes Iscia Teresinha Lopes Cendes Jamary Oliveira Filho

José Luiz Pedroso

Juliana Gurgel-Giannetti

Karina Gomes

Laura Silveira Moriyama

Lea Tenenholz Grinberg

Leandro Tavares Lucato

Leonardo Cruz de Souza

Luciano De Paola

Luciene Covolan

Luís Otávio Sales Ferreira Caboclo Marcondes Cavalcante França Jr.

Maria Fernanda Mendes

Marino Muxfeldt Bianchin

Mario Fernando Prieto Peres

Marzia Puccioni-Sohler

Michael Hornberger

Mônica Sanchez Yassuda
Octávio Marques Pontes Neto Orlando

Povoas Barsottini

Paulo José Lorenzoni

Paulo Pereira Christo

Pedro Andre Kowacs

Pedro Sampaio

Péricles Maranhão-Filho

Renato Puppi Munhoz

Rosana Cardoso Alves

Rosana Hermínia Scola

Sarah Teixeira Camargos

Sérgio Monteiro de Almeida

Sérgio Rosemberg

Sheila Cristina Ouriques Martins

Sônia Maria Dozzi Brucki

Suzana Maria Fleury Malheiros

Tarso Adoni

Vitor Tumas

Vivaldo Moura Neto

Wilson Marques Jr.

Yara Dadalti Fragoso

Ylmar Correa Neto

\section{EDITORIAL BOARD}

Acary Souza Bulle Oliveira

Alberto J. Espay

Alexis Brice

Américo Seiki Sakamoto

Andrea Slachevsky

Andrew J. Lees

Bruce Miller

Bruce Ovbiagele

Carlos Alberto Mantovani Guerreiro

Carlos Roberto de Mello Rieder

Christina Marra

Didier Leys

Fernando Cendes

Fernando Kok

Francisco Cardoso

Giancarlo Comi

Gilmar Fernandes do Prado

Henrique Ballalai Ferraz
Hugh J. Willison

Jaderson Costa da Costa

João José Freitas de Carvalho

Joaquim Ferreira

Joaquim Pereira Brasil Neto

José Manuel Ferro

Lineu César Werneck

Luiz Henrique Martins Castro

Marcelo Eduardo Bigal

Márcia Lorena Fagundes Chaves

Marco Aurélio Lana-Peixoto

Marcos Raimundo Gomes de Freitas

Maria José Sá

Maria Lucia Brito Ferreira

Marilisa Mantovani Guerreiro

Maurice Borges Vincent

Mônica Levy Andersen]

Oscar Del Brutto
Oscar Gershanik

Osvaldo José Moreira do Nascimento

Osvaldo Massaiti Takayanagui

Pedro Chaná-Cuevas

Raimundo Pereira da Silva Neto

Regina Maria Papais-Alvarenga

Ricardo Allegri

Ricardo Nitrini

Roger Walz

Rubens José Gagliardi

Sérgio Teixeira Ferreira

Stefan Schwab

Ugo Nocentini

Umbertina Conti Reed

Vladimir Hachinski

Walter Rocca 


\section{REVIEWERS}

Acary Souza Bulle Oliveira

Adalberto Studart

Adelia Maria De Miranda Henriques-Souza

Adrian Owen

Adriana Bastos Conforto

Adriana Mattos

Adriana Moro

Agustin Ibanez

Ahmet Evlice

Ailton Melo

Alberto David Fadul Filho

Alberto R.M.Martinez

Alecio Barcelos

Aleksander Erga

Alessandra Bastone

Alessandro Finkelsztejn

Alessandro Severo De Melo

Alessia Pellerino

Alexandra Prufer De Queiroz Campos

Araújo

Alexandre Alamy

Alexandre Maulaz

Alice Oliveira

Aline Kanashiro

Aline Miranda

Aline Mourao

Aline Turbino Neves Martins Da Costa

Ana Beatriz Ayroza Galvão Ribeiro Gomes

Ana Carolina Talamoni

Ana Claudia De Souza

Ana Martimbianco

Ana Mercia Fernandes

Ana Paula Fontana

Ana Paula Gonçalves

Andre Felicio

André Felipe Silva

Andre Macedo

André Santos

Andre Sugawara

Andrea Bacelar

Andrea Regner

Andrei Joaquim

Andreia Gomes Bezerra

Andreia Mendonça

Anna Paula Paranhos Miranda

Antenor Santos Silva

Antonio José Da Rocha

Antonio Lucio Teixeira

Antonio Luiz Dos Santos Werneck

Antonio-Pedro Vargas

Arlette D Doussoulin

Aroldo Bacellar

Arthur Kummer

Artur Schumacher Schuh

Audrey Nath

Ayrton Roberto Massaro

Aysel Tekeșin

Behzad Heidari

Bernardo Monaco

Bernardo Teixeira

Blener Mateus

Boris Kotchoubey

Breno Diniz

Bruno Fukelmann Guedes

Bruno Pedreira

Bruno Rodrigues

Bruno Santos-Lobato

Camila Pupe

Camilla Yamada

Caner Demir
Carina Tellaroli Spedo

Carla Matas

Carla Rynkowski

Carla Stella

Carlos Alberto Da Silva Jesus

Carlos Alberto Mantovani Guerreiro

Carlos Bernardo Tauil

Carlos Clayton Macedo

Carlos Cosentino

Carlos Henrique Camargo

Carlos Otto Heise

Carlos Roberto De Mello Rieder

Carmen Rodriguez-Blazquez

Carol Frota

Carolina Carrera

Carolina De Oliveira Souza

Carolina Moreira

Carolina Rimkus

Carolina Rouanet

Carolina Torres

Cecilia Samamé

Celal Güven

Celi Santos Andrade

Cengiz Özdemir

Christina Marra

Christos Koros

Clarissa Bueno

Claudia Ferreira Da Rosa Sobreira

Cláudia Kay

Cláudia Maia Memória

Claudia Suemoto

Claudia Szlejf

Cláudia Vasconcelos

Claudio Cunha

Claudio Scorcine

Claudio Vidal

Clecio Godeiro-Junior

Cleusa P. Ferri

Clynton Lourenço Correa

Conrado Borges

Conrado Estol

Cristiana Pereira

Cristiane Nascimento Soares

Cynthia Bedeschi

Dalva Lucia Rollemberg Poyares

Daniel Amado

Daniel Ciampi

Daniel Giansante Abud

Daniel Pimentel

Daniel Talmasov

Daniela Calainho

Daniela Rodrigues

Danielle Andrade

David Cunningham

Débora Maia

Denis Bernardi Bichuetti

Denise Cury

Denise Nicaretta

Denise Sisterolli Diniz

Deusvenir De Souza Carvalho

Didier Leys

Diogo C. Haussen

Diogo Santos

Douglas Kazutoshi Sato

Eber Corrêa

Eberval Gadelha Figueiredo

Edgard Rojas Huerto

Edmar Zanoteli

Eduardo Estephan

Eduardo Ribas
Egberto Reis Barbosa

Elcio Juliato Piovesan

Eliane Schochat

Eliasz Engelhardt

Eliseu Verly Jr

Eliza Toledo

Elizabeth Comini-Frota

Ellison Cardoso

Emine Genc

Enrico Ghizoni

Ephraim Winocur

Erasmo Barbante Casella

Eric Pasmant

Erica Tardelli

Erika Treptow

Esra Ozdil

Estela Bruxel

Esther Cubo

Eva Rocha

Evgenia Sitnikova

Eylem Tașkın

Fabiano Moulin Moraes

Fabio Porto

Fabiola Lys Medeiros

Fabiola Rachid Malfetano

Fabrício Lima

Fabrício Moreira

Felipe Oliveira

Felipe Ribeiro

Felipe Trivelato

Feres Chaddad-Neto

Fernanda Carvalho

Fernanda Goes

Fernanda Maggi

Fernanda Teresa Lima

Fernando Cendes

Fernando Figueira

Fernando Freua

Fernando Gracia

Fernando Kok

Fernando Morgadinho Coelho

Fernando Paschoal

Fernando Stelzer

Flavia Borim

Flávia Roberta Faganello-Navega

Flavio Henrique Costa

Flavio Rebustini

Francis Meire Favero

Francisca Barake Sappagh

Francisco De Assis Aquino Gondim

Francisco Dias

Francisco Eduardo Cardoso

Francisco Jose Luccas

Francisco José Montalverne

Fuad Hazime

Gabriel Braga

Gabriel Pires

Geraldo Rizzo

Gerardo Araújo Filho

Giancarlo Lucchetti

Gianfranco Spalletta

Gilberto Alves

Gildo Santos Filho

Gisela Tinone

Gisele Medeiros

Gisele Sampaio Silva

Gislaine Baroni

Giuseppina Esposito

Gloria Maria Tedrus

Gonzalo Forno 
Grace Moraes

Guilherme Abraao

Guilherme Olival

Guilherme Oliveira

Guilherme Valença

Guillermo Delgado-García

Gustavo Grottone

Gustavo Leite Franklin

Gustavo Moreira

Gutemberg Santos

Hamilton Matushita

Héctor Vargas-Pérez

Hélcio Kanegusuku

Helio Rodrigues Gomes

Hélio Yoshida

Henrique Ballalai Ferraz

Henrique Guimarães

Henry Koiti Sato

Hiteshi Chauha

Hsin Chien

Ingrid Faber

Iron Dangoni

Isabela Bensenor

Isabella Claudia Glitza Oliva

Isabella D'andrea Meira

Isabella G S De Souza

Ivan Aprahamian

Ivan Rocha Da Silva

Jamary Oliveira Filho

Janeusa Primo

Janini Chen

Jaques Waisberg

Jay Gavvala

Jay Mohr

Jefferson Becker

Jerusa Smid

Jesse Pfammatter

Jeyaraj Durai Pandian

Joan Montaner

João Amadera

João Brainer Clares Andrade

João Cação

João Eudes Magalhães

João José Freitas De Carvalho

Jonas Alex Saute

Jonas Jardim De Paula

José Alberto Landeiro

José Antonio Fiorot Jr

José Bragatti

José Cipolla-Neto

José Garbino

José Geraldo Speciali

José Luiz Cavalcanti

José Luiz Pedroso

José Manuel Ferro

José Marcelino Aragão Fernandes

Jose Otavio Soares

Juan Carlos Arango-Lasprilla

Julia Clarke

Juliana Gourlardins

Juliana Talarico Neri

Juliane Mercante

Karin Zazo Ortiz

Karla Carlos

Karolina César

Katia Lin

Kátia Vieira

Kelson Almeida

Kette Dualibi Ramos Valente

Konstantinos Tziomalos

Lara Oliveira

Laura Guilhoto

Laura Parolin
Laura Silveira-Moriyama

Laurent Cleret De Langavant

Leandro Barbosa

Leandro luamoto

Lécio Figueira Pinto

Leonardo Carbonera

Leonardo Cruz De Souza

Leonardo Giacomini

Leonardo Silva

Leticia Fezer

Leticia Sampaio

Levent Inan

Lidianne Neri

Liliana Jorge

Lineu Werneck

Lis Ferreira

Lívia Almeida Dutra

Luca Lucibello

Lucas Schilling

Lucas Scotta

Lucia Iracema Zanotto Mendonça

Luciana Branco

Luciana León Cejas

Luciane Sande

Luciano Ribeiro

Luís Dos Ramos Machado

Luís Felipe Ravic De Miranda

Luiz Carlos Marrone

Luiz Eduardo Betting

Luiz Paulo Queiroz

Luiza Piovesana

Magda Medieros

Maíra Aspahan

Manoel De Paiva Neto

Manoel Sobreira-Neto

Mansoureh Togha

Maramelia Miranda

Marcela Silagi

Marcelo Heyde

Marcelo Ribeiro Caetano

Marcelo Riberto

Marcelo Valença

Marcia Hallinan

Márcia Lorena Fagundes Chaves

Márcia Ribeiro

Márcia Silva

Marcio Balthazar

Marcio Nattan

Marco Andrey

Marco Antonio Lima

Marco Mula

Marcondes Cavalcante França Jr

Marcos Christiano Lange

Marcos Figueiredo

Marcos Gomes De Freitas

Marcos Monico Neto

Marcos Moreira

Marcos Raimundo Gomes De Freitas

Marcos Scheicher

Marcus Albuquerque

Marcus Della Coletta

Marcus Tulius Silva

Marcus Vinícius Pinto

Maria Bicalho

Maria Elisa Piemonte

Maria Isabel Freitas

Maria José Fernandes

Maria Lucia Vellutini Pimentel

Maria Montenegro

Maria Paula Foss

Maria Ravelo

Mariana Moscovich

Mariana Spitz
Marilisa Guerreiro

Marina Farah

Mario Cornejo-Olivas

Mário Emílio Dourado

Mário Fernando Prieto Peres

Mário Luciano De Mélo Silva Júnior

Marion Piñeros

Marta Imamura

Marucia Chacur

Marzia Puccioni-Sohler

Mateus Boaventura

Mauro Eduardo Jurno

Mayara Machado

Mayela Rodríguez-Violante

Mehmet Hamamci

Michel Farret

Michelle Sugimoto

Milene Rachid

Millene Camilo

Monica Koncke Fiuza Parolin

Monica Perracini

Muhtesem Gedizlioglu

Murat Alemdar

Murat Gönen

Nan Jiang

Natália Pessoa Rocha

Natalie Argolo

Nathane Braga

Nermin Tanık

Nevin Kuloğlu Pazarcı

Nicolas Farina

Niraj Kumar

Nise Alessandra Sousa

Niures Matioli

Norberto Frota

Norma Rangel

Nurhan Akaras

Octávio Pontes Neto

Orlando Graziani Povoas Barsottini

Oscar Bernal

Oscar Fernandez

Oscar Gershanik

Otto Fustes

Patricia Braga

Patricia Carvalho Aguiar

Patricia Coral

Patricia Lillo

Patricio Correa

Paula Pinho

Paulo Faro Santos

Paulo Henrique Ferreira Bertolucci

Paulo José Lorenzoni

Paulo Kimaid

Paulo Pereira Christo

Paulo Victor Sgobbi De Souza

Pedro André Kowacs

Pedro Augusto Sampaio Rocha Filho

Pedro Barbosa

Pedro Braga-Neto

Pedro Brandão

Pedro Ferreira Moreira Filho

Pedro Kurtz

Pedro Tomaselli

Péricles Maranhão Filho

Pericles Otani

Proshanto Saha

Quanguang Zhang

Rafael Carneiro

Raffaele Ornello

Raimundo Nonato Delgado Rodrigues

Raphael Castilhos

Raphael Spera

Raquel Pereira 
Raul G. Nogueira

Raul Valiente

Rechdi Ahdab

Renan Barros Domingues

Renan Castello Branco

Renata Cremaschi

Renata Ducci

Renata Guedes

Renata Kochhann

Renata Londero

Renato Marchetti

Renato Monteiro-Junior

Renato Nickel

Renato Puppi Munhoz

Ricardo Galhardoni

Ricardo Nitrini

Ricardo Orozco-Solis

Ricardo Reisin

Ricardo Silva Pinho

Rinaldo Claudino

Rita Akutsu

Roberta Arb Saba

Roberta Roiz

Roberto Prado

Robson T. Vital

Rodrigo Bazan

Rodrigo Hargers

Rodrigo Kleinpaul

Rodrigo Maia

Rodrigo Schultz

Rodrigo Secolin

Roger Brock
Roger $\mathrm{CHo}$

Roger Mello

Rogério De Rizo Morales

Ronaldo Abraham

Ronaldo Piovezan

Rosana Hermínia Scola

Rubens Gisbert Cury

Rubens José Gagliardi

Ruda Alessi

Rui Kleber Martins

Ruth Galduroz

Ruth Prieto

Ruy R R Campos

Samira Apostolos-Pereira

Sarah Teixeira Camargos

Sebastián Ameriso

Sefer Varol

Sérgio Rosemberg

Silvana Allodi

Silvia Siqueira

Sinem Sökücü

Soraia Ramos Cabette Fabio

Soraia Silva

Suzete Farias Da Guarda

Taianara Tocantins Almeida

Taissa Marinho

Tallulah Spina Tensini

Tamine Capato

Tarso Adoni

Taylor Schmitz

Taysa Bassani

Temel Tombul
Tersa Raquel Alcantara Silva

Thais Machado

Thais Massetti

Thaiza Agostini Córdoba De Lima

Theo Marins

Thiago Cardoso Vale

Tom Dening

Umbertina Conti Reed

Vaishnav Krishnan

Valeria Ferrer

Vanderci Borges

Vanessa Marques

Vasileios Kokkinos

Vera Braatz

Victor Rivera

Victoria Borba

Vitor Caldas

Vitor Tumas

Wagneer Cid

Walter Arruda

Walter Santos Moraes

Wilson Marques Jr

Wladimir Bocca Vieira De Rezende Pinto

Xian Liu

Yara Dadalti Fragoso

Ylmar Correa-Neto

Zaza Katsarava

Zeferino Demartini Jr

Zeliha Tulek

Zübeyir Huyut

Zulfi Haneef 